\title{
EUV imaging and spectroscopy for improved space weather forecasting
}

\author{
Leon Golub ${ }^{1, *}$, Peter Cheimets ${ }^{1}$, Edward E. DeLuca ${ }^{1}$, Chad A. Madsen ${ }^{1}$, Katharine K. Reeves ${ }^{1}$, \\ Jenna Samra ${ }^{1}$, Sabrina Savage ${ }^{2}$, Amy Winebarger ${ }^{2}$, and Alexander R. Bruccoleri ${ }^{3}$ \\ ${ }^{1}$ Smithsonian Astrophysical Observatory, 60 Garden Street, Cambridge, MA 02138, USA \\ 2 NASA Marshall Space Flight Center, Huntsville, AL 35812, USA \\ ${ }^{3}$ Izentis LLC, Cambridge, MA 02139, USA
}

Received 29 April 2020 / Accepted 27 July 2020

\begin{abstract}
Accurate predictions of harmful space weather effects are mandatory for the protection of astronauts and other assets in space, whether in Earth or lunar orbit, in transit between solar system objects, or on the surface of other planetary bodies. Because the corona is multithermal (i.e., structured not only in space but also in temperature), wavelength-separated data provide crucial information that is not available to imaging methods that integrate over temperature. The extreme ultraviolet (EUV) wavelengths enable us to focus directly on high temperature coronal plasma associated with solar flares, coronal mass ejections (CMEs), and shocked material without being overwhelmed by intensity from the solar disk. Both widefield imaging and spectroscopic observations of the solar corona taken from a variety of orbits (e.g., Earth, L1, or L5) using suitably-chosen EUV instrumentation offer the possibility of addressing two major goals to enhance our space weather prediction capability, namely: (1) Improve our understanding of the coronal conditions that control the opening and closing of the corona to the heliosphere and consequent solar wind streams, and (2) Improve our understanding of the physical processes that control the early evolution of CMEs and the formation of shocks, from the solar surface out into the extended corona.
\end{abstract}

Keywords: coronal mass ejection / space weather forecasts / solar corona / EUV instrumentation

\section{Introduction}

It has become clear in recent years that observations of the so-called transition corona (Masson et al., 2014), also referred to as the "middle corona", provide crucial input to forecast models via direct observation of the complex structures that affect the properties of the outflowing solar wind, and via determination of coronal mass ejection (CME) location, event onset, initial direction, path deflection, acceleration, terminal velocity into interplanetary space, shock front strength and location, magnetic field topology, rotation and reconnection of field lines, mass and temperature of CME components, among other observed parameters (Temmer, 2016). Forecast models are especially sensitive to initial input values (Riley et al., 2018), implying that early measurement of these values will improve the models (Hinterreiter et al., 2019).

In the next section we discuss some salient features of the corona and of coronal observation in the extreme-ultraviolet (EUV). We then discuss some of the instrumentation and

\footnotetext{
*Corresponding author: lgolub@cfa. harvard. edu
}

methods available for improving coronal observations with the goal of achieving more accurate and reliable space weather forecasts.

\section{Coronal EUV observations}

With the development in the 1970 s of precision multilayer coatings having sharp boundaries between layers and $d$-spacing small enough to reflect EUV and soft X-ray photons at normal incidence (Spiller, 1974; Haélbich \& Kunz, 1976; Barbee, 1985), it became clear that microscopes and telescopes could be made for imaging the short wavelengths in the 44-400 wavelength range (Catura \& Golub, 1988; Golub et al., 1989). The necessary refinements in substrate polishing and figuring, and the lengthy testing needed to find suitable stable and smooth (at the atomic scale) material combinations, were motivated in large part by EUV lithography for next generation lithography, but applications in astronomy - especially for solar observation - soon followed (Bruner et al., 1988; Walker et al., 1988; Golub et al., 1990). 


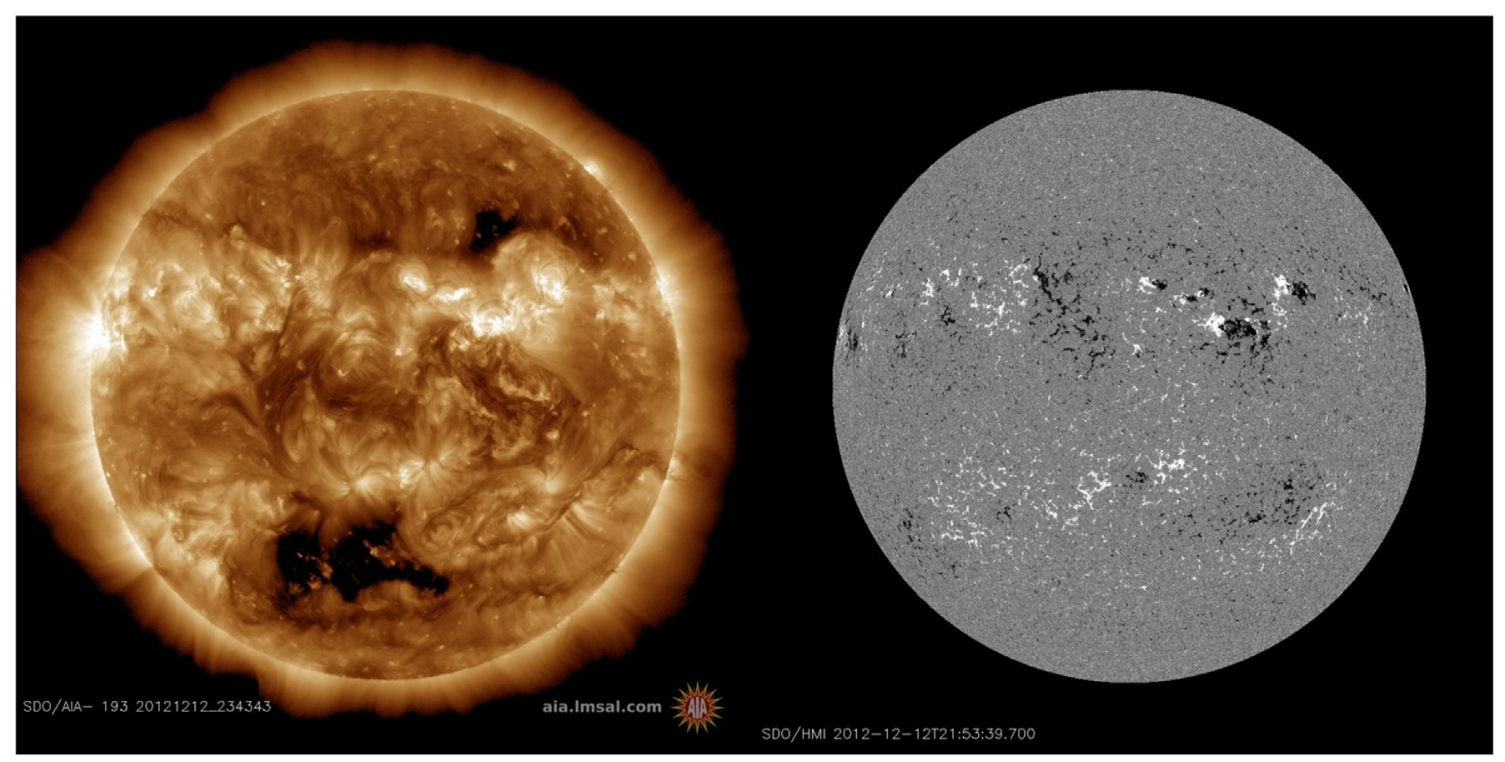

Fig. 1. 12 December 2012; (left) $193 \AA$ passband image from SDO/AIA showing the magnetically-dominated coronal EUV emission, and (right) the surface magnetic field from the SDO/HMI.

The narrowband nature of normal incidence EUV multilayer coatings is turned to advantage by centering the multilayer passband on a strong coronal emission line. The reflectance is then, to a great extent, dominated by emission from coronal plasma at the temperature of formation of the ion in question. Figure 1 shows an image of the solar corona centered on the Fe XII emission line at $195 \AA$, along with an image of the surface magnetic field strength and location from the Solar Dynamic Observatory's (SDO) Atmospheric Imaging Assembly (AIA) and Helioseismic and Magnetic Imager (HMI) instruments. The close connection between the locations of strong surface fields and hot coronal emission has long been established (e.g., Golub \& Pasachoff, 2009, Ch. 6), and this figure also illustrates the complex coronal topology that accompanies the emergence and evolution of the coronal magnetic fields.

\subsection{The multithermal corona}

On-disk X-ray and EUV observations soon made clear that the corona is multithermal (Walker et al., 1993). The corona's appearance differs markedly at distinct wavelengths corresponding to spectral emission lines formed at various temperatures, as illustrated in raster images obtained with the Hinode EUV Imaging Spectrometer (EIS; Fig. 2). Observations of the solar corona can thus be thought of as at least five-dimensional: it is highly structured in volume, varies with time, and is temperature-dependent, with discrete structures visible at different coronal temperatures. Ideally, one would want high spatial resolution observations over a wide field of view, at high temporal cadence, and at multiple EUV wavelengths simultaneously in order to properly characterize high-temperature coronal features.

The need for such data is especially acute for the detection of CMEs, which move outward from the visible surface rapidly and are comprised of hot and cool components. The visibility of hot versus cool portions of the CME is highly variable from event-to-event (Fig. 3). In addition to aiding in the detection of these events, measuring the partition of energy among these components is useful for determining the location of and mechanisms leading to the production of solar energetic particles (SEPs) (Reames, 1999).

\subsection{The transition corona}

The highly dynamic interface region lying between the low- $\beta$ inner corona (dominated by closed magnetic structures) and the high- $\beta$ outer corona (consisting largely of radial features) represents the inverse of the chromosphere-corona transition region at the base of the corona (Masson et al., 2014). This transition is highly variable in time and may occur anywhere from 1.3 to $>3 R_{\odot}$ (Vásquez et al., 2003), or even farther out at high latitudes. DeForest et al. (2016) argue, based on the change in character of the observed streamer belt plasma outflow, that a transition occurs $10^{\circ}-20^{\circ}$ out. Such a transition or transitions define the crucial boundary that allows hot magnetized plasma to escape from the corona into interplanetary space, acting as either a pathway or an obstacle to solar wind streams and eruptive events as they leave the Sun.

While the potential field source surface (PFSS) gives a general idea of the connectivity in the unexplored middle volume, the reality is that the field is much more complex there, and structures do not generally smoothly transition from closed to radially open. Many structures are non-radial, like coronal fans (Seaton et al., 2013). The build-up of magnetic energy in the closed field can also alter the shape of the open field, displacing the streamers and pseudostreamers from their potential configuration. Even a minor displacement can alter the expansion factor of open magnetic fields, thereby affecting the solar wind speed or deflecting erupting material in an unexpected manner.

Many theoretical and numerical studies have explored the connection between the heliosphere and the corona, but the difficulty lies in matching these models to observations. 


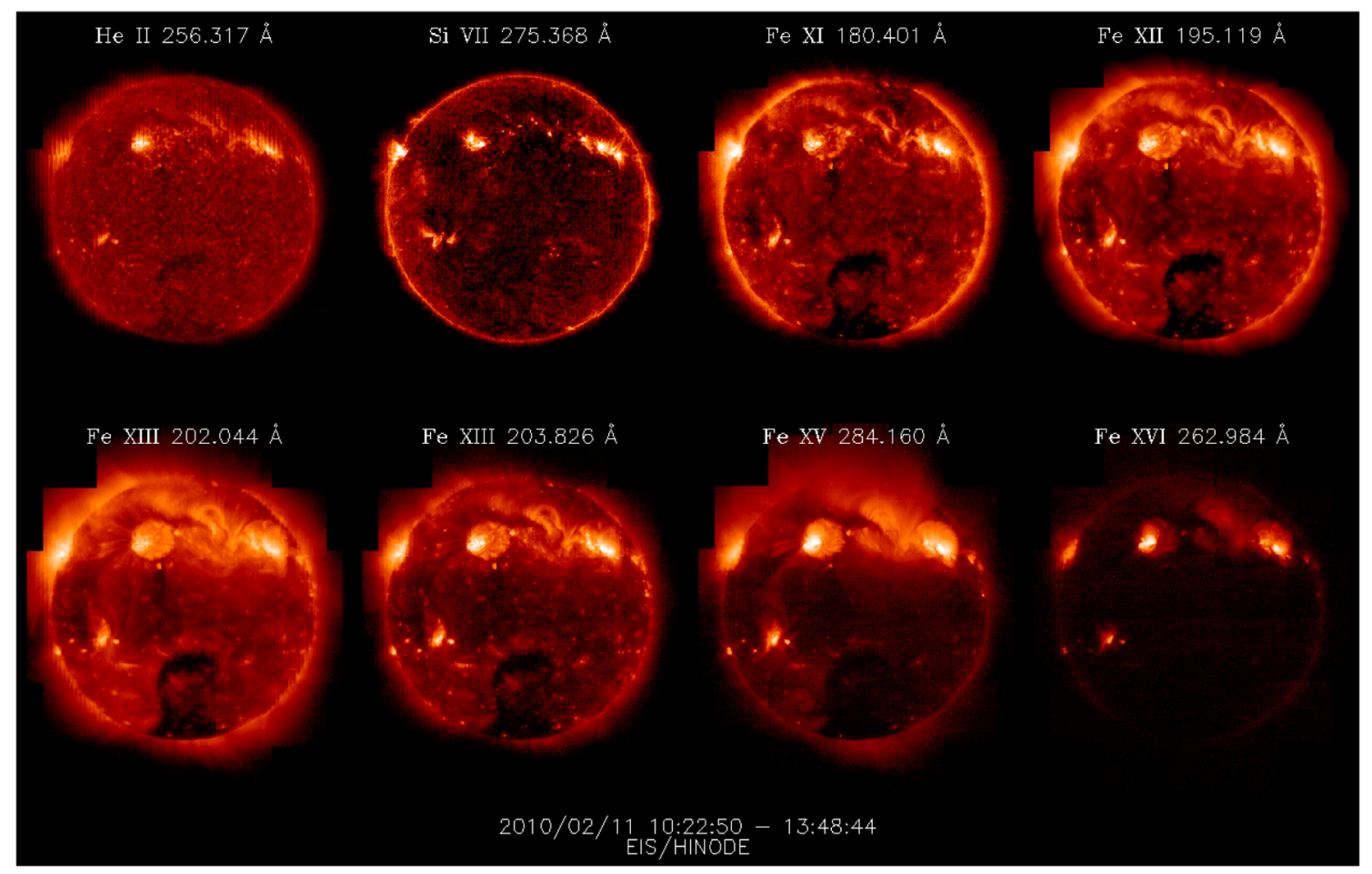

Fig. 2. Hinode/EIS produced a mosaic of slot images on 11 February 2010, to form a series of full disk images in spectral lines formed at temperatures ranging from $<10^{5} \mathrm{~K}$ (He II) to $\sim 3 \times 10^{6} \mathrm{~K}$ (Fe XVI), thereby illustrating the multithermal structuring of the EUV corona. (Photo credit: JAXA/NASA/ESA Hinode/EIS).

The S-Web model (Antiochos et al., 2011), for instance, predicts that a lattice of separators and quasi-separatrix layers permeates the corona. This network marks the locations where plasma in closed magnetic structures gains access to the solar wind via reconnection with open field lines, which should leave imprints in the EUV corona. The web (imaged from magnetohydrodynamic (MHD) models) only becomes separable from the highly structured closed field corona at heights where the solar wind begins to dominate the magnetic field $\left(\sim 2 R_{\odot}\right.$ and above). A wide-field imager capable of imaging EUV plasma at larger heights (Del Zanna et al., 2018) provides a unique opportunity to test the S-Web model (Riley et al., 2011).

Intermittent interchange reconnection between closed and open magnetic fields has been proposed as the source of the variability of the slow solar wind, parts of which have exhibited closed field plasma characteristics at 1 AU (Fisk, 2005; Del Zanna et al., 2011; Pontin \& Wyper, 2015; Weberg et al., 2015). It is strongly debated whether the open field associated with slow solar wind is mixed within closed-field regions (Fisk $\&$ Zurbuchen, 2006) or whether it is confined to arc-like bands at quasi-separatrix surfaces that mark the open/closed field boundaries (S-web model). In the Wang-Sheeley-Arge (WSA) model (viz. Sheeley, 2017), the wind speed is highly dependent on the non-radial expansion of the field near the surface. Hence, fast wind originates from open-field $\mathrm{CHs}$ and slow wind from near open/closed boundaries. All of these models predict that the slow wind originates where open and closed fields meet, but the topology of that boundary is an important factor that will help to determine what best captures the slow solar wind structure.

\subsection{EUV input to space weather forecasts}

The ability to observe the EUV corona off-limb and with high sensitivity led to the realization that the emission extends substantially farther out than was expected, to a radial distance of at least 2.5-3 $R_{\odot}$ (Slemzin et al., 2008). Although the extended emission in coronal streamers is now regularly observed (Tadikonda et al., 2019), the explanation for this elongated visibility is still under discussion (Goryaev et al., 2014). Nevertheless, it is now well established that loop and streamer structure is seen in the EUV corona out to at least $3 R_{\odot}$. The corona in this region is extremely dynamic and variable on time scales from minutes to hours, permitting detection of CMEs in the EUV out to the distance at which white light coronagraph observations typically begin.

Central to the understanding of solar and space physics is knowledge of the connection between the solar corona and the heliosphere. The heliospheric magnetic field is composed of an extended open field anchored in the photosphere. This field and associated plasma are diverted from a purely radial direction by currents that produce a complex magnetic topology, which is determined by photospheric evolution, prior dynamic events, and the field's global structure (Newkirk et al., 1968; Wang, 1996; McComas et al., 2007; Yeates et al., 2008; Mozer et al., 2020).

Tracing the path of an event that moves past an in situ sensor to its solar origin is highly dependent on the intervening plasma and field. The boundary between the open and closed field fluctuates and is distorted by physical processes in the corona on a broad range of scales (e.g., magnetic reconnection, 

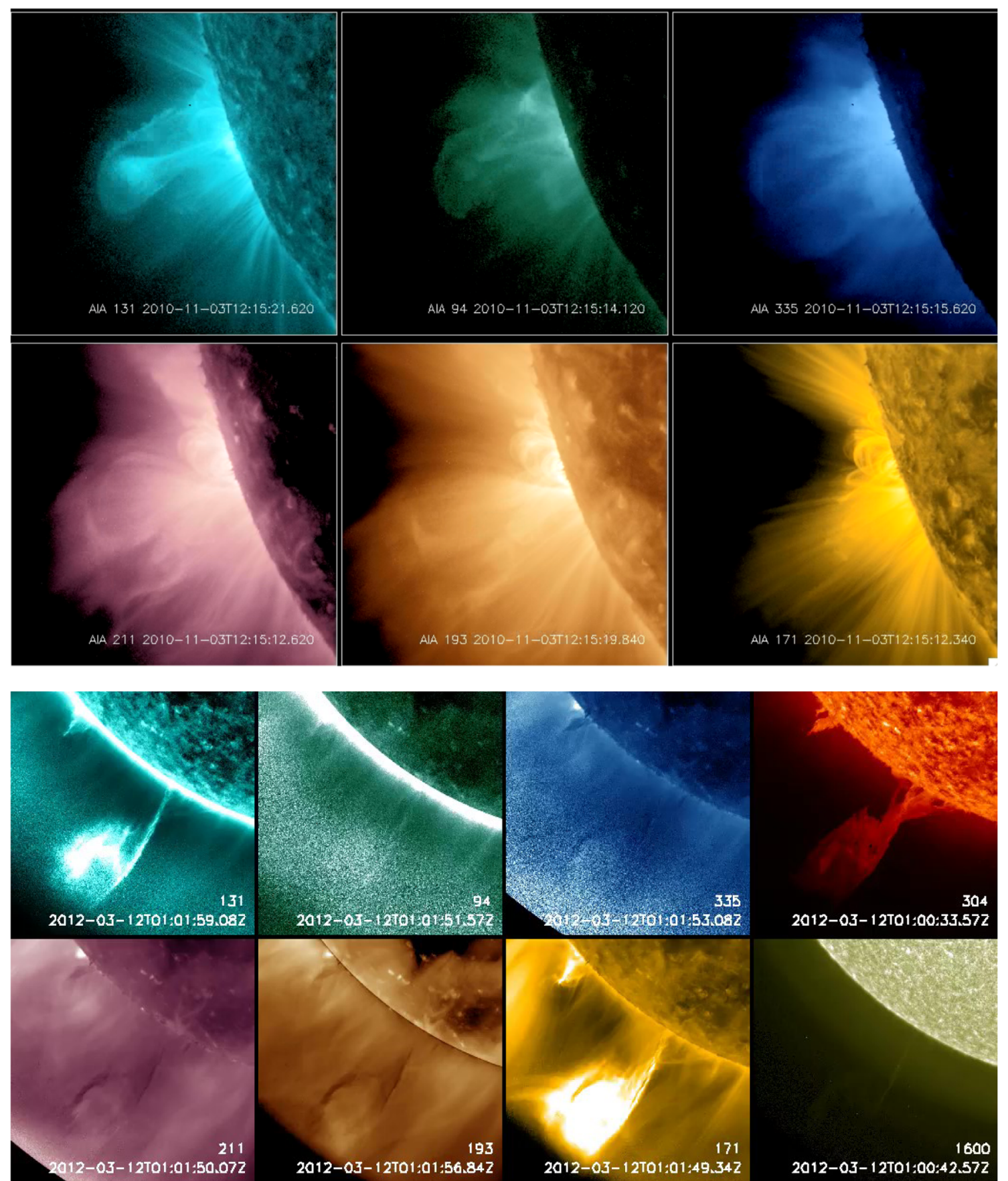

2012-03-12T01:00:33.572
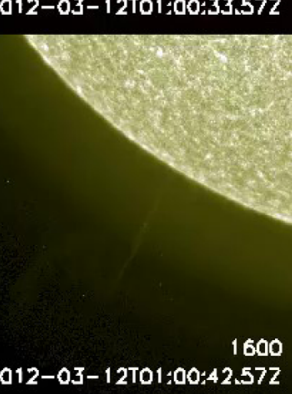

Fig. 3. CMEs are multi-thermal with their primary features appearing in either hot or cool lines. Top panel: A hot CME is bright in the $335 \AA$ (Fe xVI; 3 MK), $94 \AA$ (Fe xVIII; $~ 6 \mathrm{MK}$ ), and $131 \AA$ (Fe xxI; >10 MK) channels, but nearly invisible in the cooler lines (Reeves \& Golub, 2011). Bottom panel: A cool CME is seen clearly in the $304 \AA$ (He II; $~ 50,000 \mathrm{~K}$ ) channel and in $171 \AA$ (Fe IX; 0.6 MK), but only in absorption in Fe XII-XVI ( 1-3 MK). In the $131 \AA$ channel, the CME is visible due to emission from Fe vIII (Su et al., 2015). Observations must detect plasma over this wide range of temperatures and be able to separate the components spatially.

eruptions, and continual flux emergence; Abbo et al., 2016). The feedback between these processes and the open/closed transition boundary is poorly understood, largely due to a lack of sensitivity and coverage in this region. Understanding this feedback is critical for heliospheric studies since it determines how hot magnetized plasma enters interplanetary space.

\subsubsection{Early onset of solar eruptions: coronal dimmings and EUV waves}

The regions in the solar atmosphere where concentrations of these open field lines are rooted can become rapidly evacuated of plasma and are subsequently termed transient coronal 


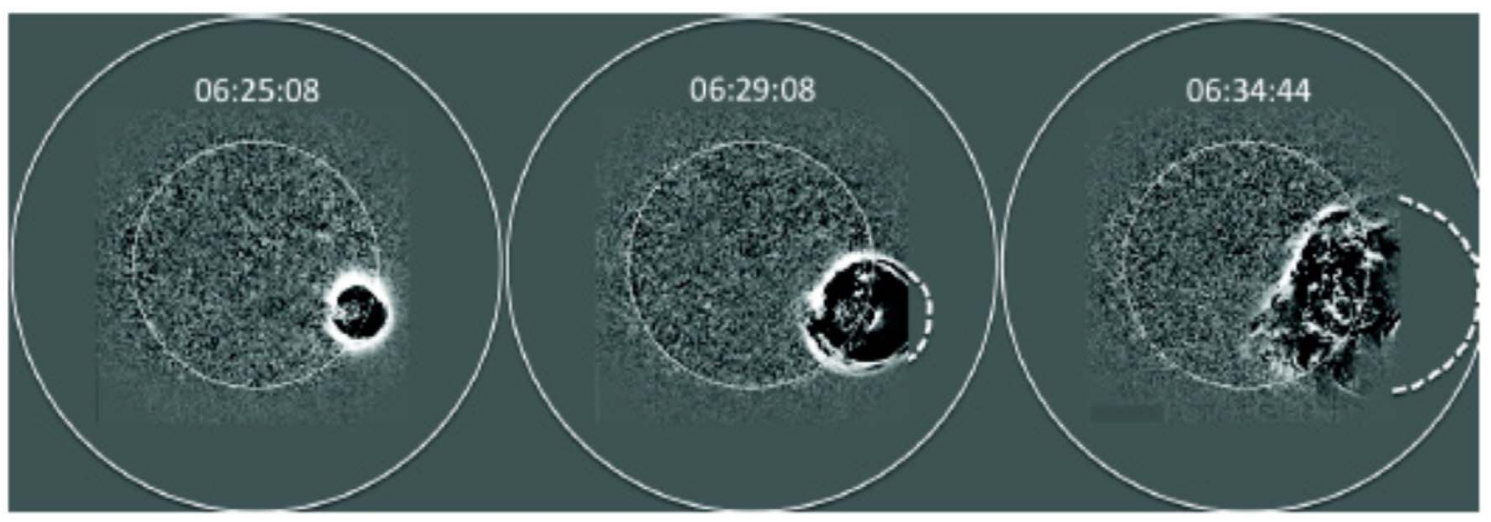

Fig. 4. Three AIA $193 \AA$ base-difference images showing the evolution of an off-limb wave. The data were binned to $4.8^{\prime \prime} /$ pixel. The outer (solid) circle is $2 R_{\odot}$, and the dashed lines indicate the extrapolated portions of the wave outside of the AIA FOV.

holes (CHs) or coronal dimmings. These transient reductions in intensity represent a significant low-corona signature of a CME (Sterling \& Hudson, 1997) and when observed on-disk are an indicator of an Earth-directed CME eruption (Thompson et al., 2000). A careful analysis of the dimming regions can provide early determination of critical energy transfer properties, such as the mass and peak velocity of the departing CME (Robbrecht \& Wang, 2010; Dissauer et al., 2018, 2019; Veronig et al., 2019).

Recent work has suggested that shocks and blast waves caused by CMEs and flares can energize ions and electrons up to SEP energies of tens of $\mathrm{MeV}$, even below $5 R_{\odot}$ (Veronig et al., 2010; Dresing et al., 2012; Battarbee et al., 2013; Kozarev et al., 2013). Shocks may be driven if the CME exhibits significant over-expansion, or if it enters regions of decreased Alfvén or fast magnetosonic speed. Such regions are expected above many ARs, as well as in QS regions (Evans et al., 2008; Zucca et al., 2014). High-resolution, multi-band EUV imaging, particularly from the SDO/AIA, has proven to be an excellent tool to study detailed morphology and kinematics of shocks, as well as key shock parameters including the compression ratio (Gopalswamy \& Yashiro, 2011; Ma et al., 2011; Bein et al., 2012; Kozarev et al., 2015).

Figure 4 shows an example of a coronal shock observed by AIA. The expanding shock feature is evidenced by the appearance of an abrupt jump in the EUV intensity during the shock passage.

\subsubsection{CME trajectories}

CMEs begin their acceleration low in the corona, and many events experience their peak acceleration below $0.5 R_{\odot}$ (Bein et al., 2011). Thus coronagraphs miss the initial CME acceleration phase in many cases. EUV instrumentation has been used to fill the gap (e.g. Gallagher et al., 2003), but it can be difficult to accurately and fully derive CME kinematics because of the mismatched fields of view and wavelength ranges of the instrumentation that is used to observe CMEs. An exception came in the early days of SoHO, when Zhang et al. (2001) used the C1 instrument on LASCO (with a field of view of 1.1-3 $R_{\odot}$ ) to derive the temporal relationship between flare emissions and CME acceleration. More recently, Veronig et al. (2018) used the wide-field SUVI instrument on the GOES satellite to track the CME front produced in the September 102017 event from the low corona into the LASCO field of view. These rare examples show the utility of spatially continuous observations beginning low in the corona.

\subsubsection{Stealth CMEs}

Before the launch of the twin STEREO spacecraft it was widely accepted that Earth-directed CMEs will display some of the above-mentioned low coronal signatures, or other signatures such as a long-duration X-ray flare, a filament eruption, or post-flare loops (Hochedez et al., 2005). Statistical studies consistently found that the correlation between any given indicator and a geo-effective CME is often quite poor, leading Robbrecht et al. (2009) to provide the first clear indication that some CMEs, visible in EUV images, have no clear low coronal signature. These events were designated "stealth CMEs" and the paper concluded that "Our analysis thus strongly suggests that reliable prediction of Earth-directed CMEs can only be made by remote-sensing platforms away from the Sun to Earth line." A statistical study of CMEs during solar minimum found that one out of every three CMEs is a stealth CME (Ma et al., 2010). While there were no flare loops or EUV waves present in the coronal observations for these events, 8 out of 11 stealth CMEs did exhibit some sort of coronal structure off-limb within the STEREO EUVI field of view. A subsequent study of stealth CMEs from 2012 determined that stealth CMEs are somewhat slower than events with an obvious low-coronal signature, and that they often originate near the north pole (D'Huys et al., 2014). Alzate \& Morgan (2017) re-analyzed the stealth CMEs examined by D'Huys et al. (2014) and found that low coronal signatures could be revealed in the EUV observations with further image processing. EUV instrumentation can thus be useful for identifying the source regions for these events.

Events without obvious low coronal signatures can be important for space weather. Stealth CMEs have been shown to be be geo-effective depending on the accompanying highspeed solar wind and a stealth CME caused the third-largest geomagnetic storm of Solar Cycle 24 (Mishra \& Srivastava, 2019). An imager that can connect clear signatures of CMEs in coronagraphs to source regions in the low corona will be a great asset to space weather prediction. 


\subsubsection{Energization of coronal mass ejections}

Magnetic reconnection is widely expected to trigger and energize dynamic flaring events, releasing significant amounts of energy into the corona in a matter of seconds. Reconnection, however, cannot be directly observed due to lack of magnetic field observations in the corona at rapid temporal resolution. Consequently, indirect observational signatures of reconnection have been studied extensively in the lower corona for decades, successfully providing insight into energy release mechanisms. EUV observations have been used to diagnose flows (e.g. Yokoyama et al., 2001; Savage et al., 2012b; Su et al., 2013), topology (e.g. Sun et al., 2015), and the temperature structure (e.g. Hannah \& Kontar, 2013; Yan et al., 2018) of regions where reconnection is likely occurring. Supra-arcade downflows (SADs) and shrinking loops are particularly useful features as they may indicate the presence and path of retracting postreconnection flux (Savage et al., 2010, 2012a). Observing these reconnection outflow candidates is a challenge due to low signal-to-noise in the pertinent regions of interest in the outer corona.

To constrain reconnection models, it is necessary to observe eruptions near their point of origin. Downflows that could be related to reconnection outflows have been observed many hours after the passage of CMEs through the FOV of the white light coronagraph on board the SoHO (e.g., Sheeley \& Wang, 2007). An event reported by West \& Seaton (2015) shows clear detection of these downflows high in the EUV corona for at least a week after the initial eruption. The dynamics of these features through the extended solar atmosphere is clear, but obtaining unambiguous diagnostics is not currently possible due to limited cross-calibration and the difference in operational parameters (i.e., wavelength, resolution, exposure time) between currently available instruments.

A wide-field EUV imager with high dynamic range capability is ideal for observing and analyzing these critical signatures of continual energy release behind CMEs, as it is vital to: (1) track them from their point of origin high in the corona down to their footpoints; (2) detect them in the low-signal environment of the outer corona as well as near the bright disk with the same exposure setting; and (3) observe them with a consistent wavelength bandpass using a single system to avoid cross-calibration issues.

\section{The COSIE instrument}

Slitless spectrographs for solar coronal imaging have been used for many years (e.g., Tousey et al., 1973; Silk et al., 1975; Zhitnik et al., 1998). Here we discuss a next-generation instrument, the COronal Spectrographic Imager in the EUV (COSIE), that improves upon the successful Skylab S-082A instrument via an extension to shorter wavelengths, and the RES-K instrument via a greatly improved reflection grating that minimizes image distortion. The COSIE instrument utilizes recent advances in EUV instrument design to produce a twochannel instrument that allows for the tracking and analysis of coronal events from the solar disk to $>3 R_{\odot}$.

The COSIE-C wide-field imaging optical system consists of an entrance aperture, a flat fold optic, an f/6.7 focus mirror, and a detector. Light reflects off the flat feed mirror, to a spherical focus mirror and back onto the detector. In the COSIE-S slitless spectrograph system, the flat feed optic is replaced by a blazed reflection grating.

\subsection{Widefield imaging channel}

The dual-channel COSIE design is shown in Figure 5. The flat mirror feeding COSIE-C has a 4 layer-pair $\mathrm{Zr} / \mathrm{Al}$ multilayer coating with an EUV reflectance of $>60 \%$ over the full COSIE wavelength range. The focus mirror coating is similar to the $\mathrm{Mo} / \mathrm{Si}$ multilayer used on the Hinode, with 10 layer-pairs instead of 20 , producing a broader response with a slight decrease in peak reflectance and very high throughput at the focal plane. The multilayer coatings enable EUV imaging and have been used in many solar missions, including the Solar and Heliospheric Observatory's (SoHO) EUV Imaging Telescope (EIT), the Transition Region and Coronal Explorer (TRACE), Hinode/EIS, and SDO/AIA.

This channel can take advantage of a back-illuminated CCD camera with an apodized focal plane filter - one with a disk having higher absorption covering the solar disk and the brightest inner corona - to allow for simultaneous recording of the bright inner corona near $1 R_{\odot}$ and the fainter outer coronal structures at $\sim 3 R_{\odot}$ within the same image. An active pixel CMOS camera with controlled readout of faint vs. bright pixels can also be a suitable detector option.

COSIE-C is a high-sensitivity broad-band imager in the 186-205 $\AA$ range optimized to observe slowly evolving streamers, pseudo-streamers, and coronal hole $(\mathrm{CH})$ boundaries as well as dynamic solar eruptive events. A single exposure captures on and off disk plasma out to $3.3 R_{\odot}$, and a low noise $\left(<25 \mathrm{e}^{-}\right)$camera enables imaging of faint structures with exposure times of $0.1-3 \mathrm{~s}$ and an image cadence of 6-12 s (Del Zanna et al., 2018).

\subsection{Slitless spectrograph imaging channel}

The COSIE-S feed-optic (grating) is a blazed 5000 lines $/ \mathrm{mm}$ grating operating in 2nd order, sending the dispersed EUV light onto the same focus optic used for the $-\mathrm{C}$ channel. The grating performance is controlled by the grating surface quality, its blaze, and the coating applied to the surface. To produce a grating with the necessary surface finish and blaze angle, we use a process of nano-fabrication developed at the Massachusetts Institute of Technology (MIT) (Chang, 2004), with improvements described in Section 3.2.2.

Slitless spectrometers can provide simultaneous imaging and spectral data over an extended field of view, thereby allowing rapid data acquisition for extended sources. In COSIE-S, spatially resolved full disk solar images are formed at multiple wavelengths, and partially overlap on the detector. The horizontal dimension of the focal plane contains both spectral and spatial information (Fig. 6). The grating angle of incidence compresses the scale in the dispersion direction, so that the solar disk maps to an ellipse with a 1:3 aspect ratio.

The strongest lines in the COSIE passband are listed in Table 1, along with some additional lines that tend to be weaker but are density-sensitive at coronal temperatures. The list shows typical expected count rates for a range of coronal features, and indicates that a broad range of temperatures from $5.35<$ $\log T<7.25$ is covered within this $20 \AA$-wide bandpass. 


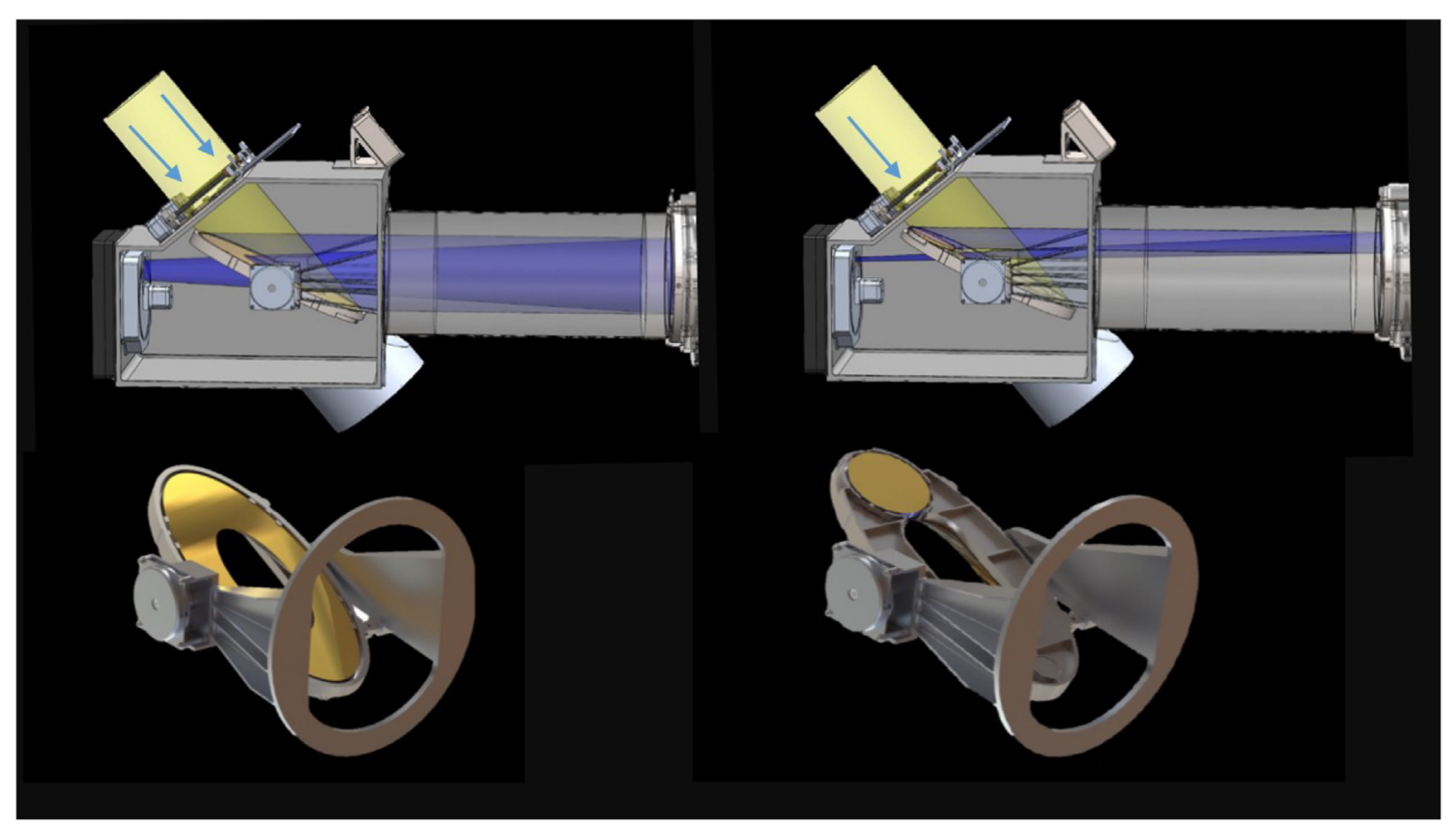

Fig. 5. The dual-channel COSIE design uses a rotating flip mechanism to select between the wide-field imager COSIE-C channel (left) and the slitless spectrograph COSIE-S channel (right). Solar EUV enters from the upper left, as indicated by the blue arrows, with visible light blocked by a thin $\mathrm{Al}$ entrance filters. Light then reflects from the rotatable flip mechanism selector onto a multilayer-coated focusing optic that is alternately used by both channels.

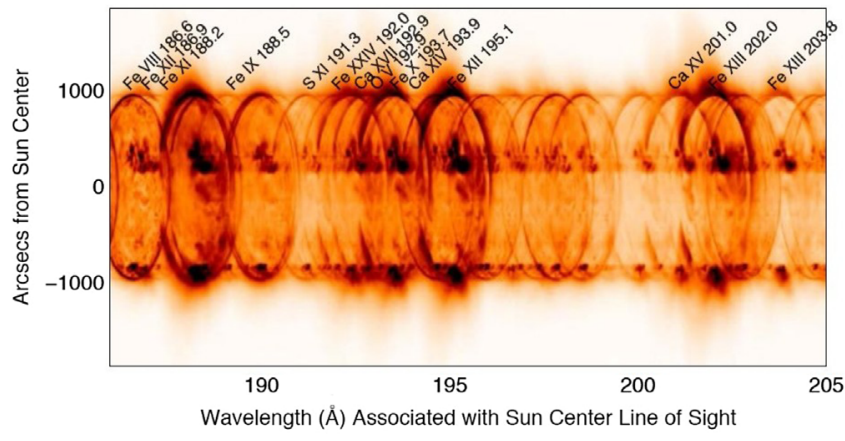

Fig. 6. A simulated image of the slitless spectrograph channel, based on SDO/AIA data, shows the full-disk solar images produced by this instrument. The contracted plate scale in the dispersion direction is due to the off-normal angle of incidence employed for higher spectral resolution.

\subsubsection{Wavelength separation}

To extract temperature and density information from COSIE-S, an analysis tool to separate the wavelength contributions in overlapping portions of the image may sometimes be needed. Because the solar images between 186 and $205 \AA$ are offset according to the instrumental dispersion, there can be a mixing of spatial and spectral information within the overlap. To accomplish this separation, techniques have been developed to distinguish spatial and spectral information (Cheung et al., 2019; Winebarger et al., 2019). ${ }^{1}$ While COSIE-S has sufficient

\footnotetext{
${ }^{1}$ A similar difficulty arises with multi-slit spectrographs, such as MUSE (De Pontieu et al., 2020) which can be inverted in a similar way.
}

Table 1. Strong lines in the COSIE-S wavelength range.

\begin{tabular}{lcccc}
\hline Ion and & \multirow{2}{*}{$\begin{array}{c}\text { Log maximum } \\
\text { wavelength }(\AA)\end{array}$} & \multicolumn{2}{c}{ Expected signal $\left(\mathrm{ph} \mathrm{s}^{-1}\right)$} \\
\cline { 3 - 5 } & temperature & QS & AR & Flare \\
\hline O v 192.91 & 5.35 & 0.7 & 3.6 & 2120 \\
O iVI 184.12 & 5.45 & 0.6 & 7.4 & 500 \\
Fe VIII 185.21 & 5.65 & 10.9 & 102 & 4460 \\
Fe IX 188.50 & 5.85 & 10.1 & 104 & 1760 \\
Fe X 184.54 & 6.05 & 18.8 & 234 & 2800 \\
Fe XI 188.22 & 6.15 & 30.4 & 577 & 6570 \\
Fe XII 195.12 & 6.20 & 30.8 & 1135 & 14,355 \\
Fe XII 186.88 & $6.20^{\mathrm{a}}$ & 8.7 & 304 & 3800 \\
Fe XIII 202.04 & 6.25 & 5.8 & 548 & 8620 \\
Fe XIII 203.83 & $6.25^{\mathrm{a}}$ & 4.0 & 355 & 5520 \\
S XI 191.27 & 6.30 & 0.5 & 50 & 960 \\
Ar XIV 194.39 & 6.55 & 0.0 & 8.1 & 1130 \\
Ca XIV 193.87 & 6.55 & 0.0 & 20.6 & 3500 \\
Ca XV 200.97 & 6.65 & 0.0 & 13.6 & 4840 \\
Ca XVII 192.86 & 6.75 & 0.0 & 26.2 & 62,200 \\
Fe XXIV 192.04 & 7.25 & 0.0 & 0.0 & 80,690 \\
\hline
\end{tabular}

${ }^{a}$ Density-sensitive line.

The final three columns give the signal in the COSIE-S channel for three standard Chianti differential emission measures, assuming a density of $10^{9} \mathrm{~cm}^{-3}$, under quiet sun, active region, and flare conditions folded through the COSIE-S effective area. Intensities are calculated using the solid angle associated with a COSIE-S pixel and integrated over the spectral line.

resolution to discern Doppler shifts and line broadening, the inversion method discussed here is designed for robustly separating the entire spectrogram using a resolution of $\sim 0.01 \AA$. Note that many events, such as compact flares and bright isolated active regions, will not have substantially overlapped 

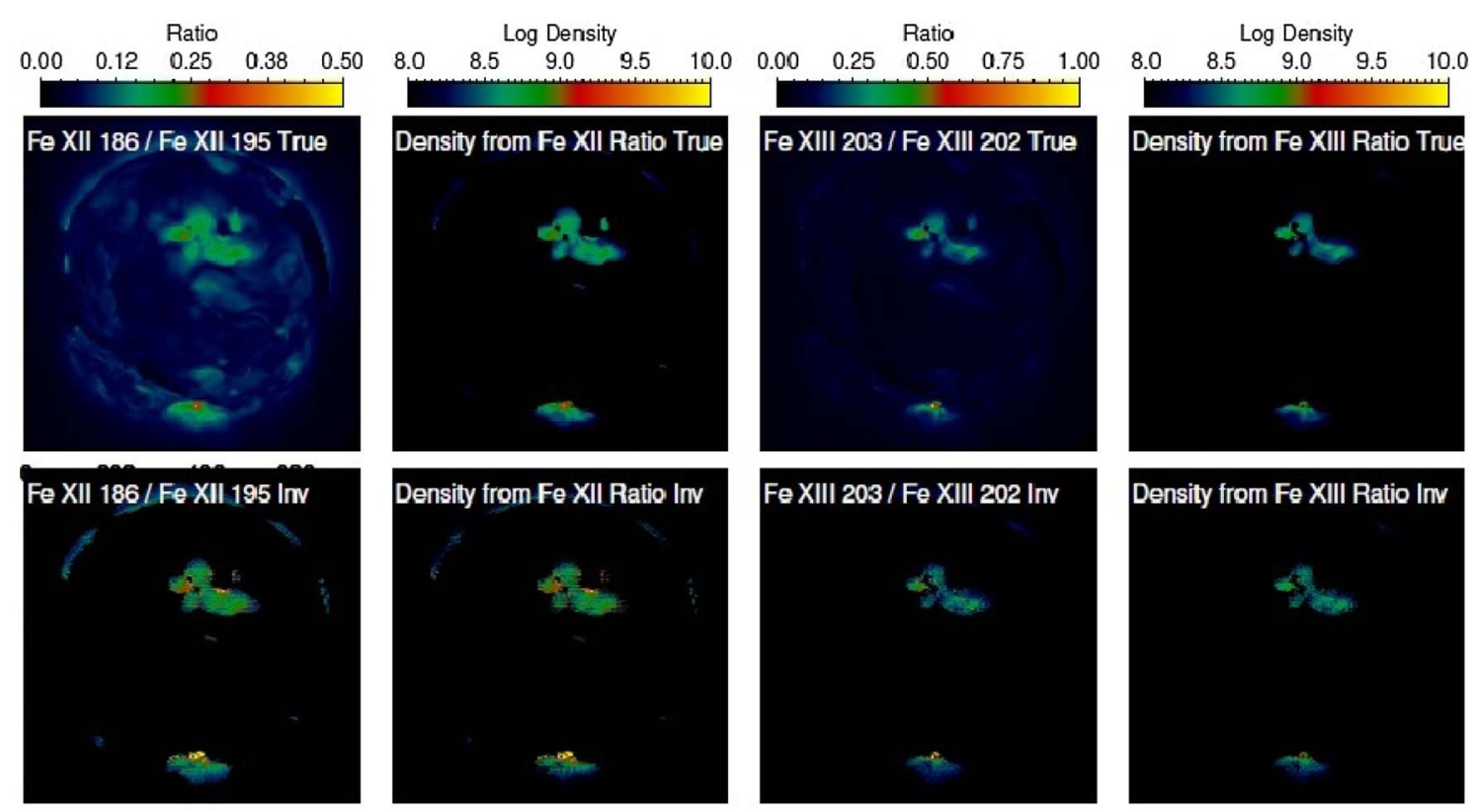

Fig. 7. Results of the wavelength unfolding via inversion. Comparison shows the full Sun ratio of the Fe XII and Fe XIII lines from truth data (top row) and from inverted data (bottom row), as well as the coronal density determined from the Fe XII and Fe XIII ratios.

spectra. In these situations, the spectral and spatial features can be decoupled without further processing.

The inversion method utilized to unfold COSIE data is a sparse inversion, which closely follows the method developed by Cheung et al. (2015) that has been shown to successfully reproduce thermal diagnostics from SDO/AIA data. The method not only minimizes the $\chi^{2}$ but also the sum of the emission measure matrix multiplied by a weighting coefficient; this additional term makes this algorithm a sparse inversion. The first step is to write the data vector as the multiplication of the response matrix times an emission measure vector. The response matrix and emission measure vector are generally taken to be functions of temperature, as in Cheung et al. (2015), but can be expanded to include velocity and density if the observations provide adequate discrimination over those parameters.

The code is written in the interactive data language (IDL) but calls the Python LASSOLARS subroutine that completes the minimization via the IDL-to-Python bridge. Because both spatial and spectral information are folded together in the spectrograph data, the inversion is completed on a single row of the $\mathrm{S}+\mathrm{C}$ data at a time, meaning the emission measure is determined for each spatial location along a row simultaneously. Inversion takes 0.1-20 s per row, depending on the resolution and number of free parameters, on a Mac laptop with a $2.9 \mathrm{GHz}$ Intel Core i9 processor and 16 GB RAM. The inversion is completely parallelizable; each row can be calculated independently. Comparisons of the inverted maps to the truth data are shown in Figure 7. More details are available in Winebarger et al. (2019) which also discusses in detail the implications of the count rates in the various coronal features listed in Table 1 , including the camera properties such as QE and noise.

\subsubsection{Diffraction grating}

The spreading of the wavelengths in the COSIE-S channel is accomplished via a feed optic that is a blazed 5000 lines $/ \mathrm{mm}$ grating operating in 2 nd order. To produce a grating with the necessary surface finish and blaze angle, we use a process of nano-fabrication developed at MIT (Chang, 2004) with improvements described below. The system utilizes a property of the silicon crystal that makes certain crystal faces selectively less reactive to potassium hydroxide $(\mathrm{KOH})$. Specifically the etch rate of the $\{111\}$ planes are $\sim 100 \times$ slower than the non$\{111\}$ planes. The silicon substrate is $\langle 111\rangle$ crystal orientation, custom off-cut at the desired blaze angle. The silicon wafer is coated with thermal $\mathrm{SiO}_{2}$, which is then patterned to the designed grating-period via interference lithography. The thermal $\mathrm{SiO}_{2}$ layer is reactive-ion etched and the wafer is then immersed in $\mathrm{KOH}$. The thermal $\mathrm{SiO}_{2}$ masks the grating lines as the spaces are etched away until they hit the angled $\{111\}$ planes. The process then applies repeated RCA-1/HF to remove any nubs and roughness from the $\mathrm{KOH}$ etching. The result is a blazed grating.

The COSIE flight grating is currently targeted to be a single grating, on a $90 \mathrm{~mm}$ diameter, thick single crystal silicon substrate. The period is $200 \mathrm{~nm}$ with a sawtooth angle of $13.25^{\circ}$. The target resolution is 5000 lines $/ \mathrm{mm}$. We have currently demonstrated the ability to fabricate $200 \mathrm{~nm}$ pitch gratings on silicon wafers with a $13.25^{\circ}$ sawtooth (Fig. 8).

\subsubsection{Grating coating}

We predicted the optimal grating coating using PCGrate, a proprietary grating efficiency solver (Goray, 2005). PCGrate uses an optical integral method to numerically calculate absolute efficiencies across all diffraction orders. It provides a framework for the iterative optimization of diffraction grating efficiency by parameterizing a number of geometric and material properties and allowing them to vary during calculation. Our opitmization efforts focused on the 2nd-order grating efficiency, which took place in three stages: (1) determining the optimal coating material, (2) modeling the effectiveness of a multilayer configuration, 


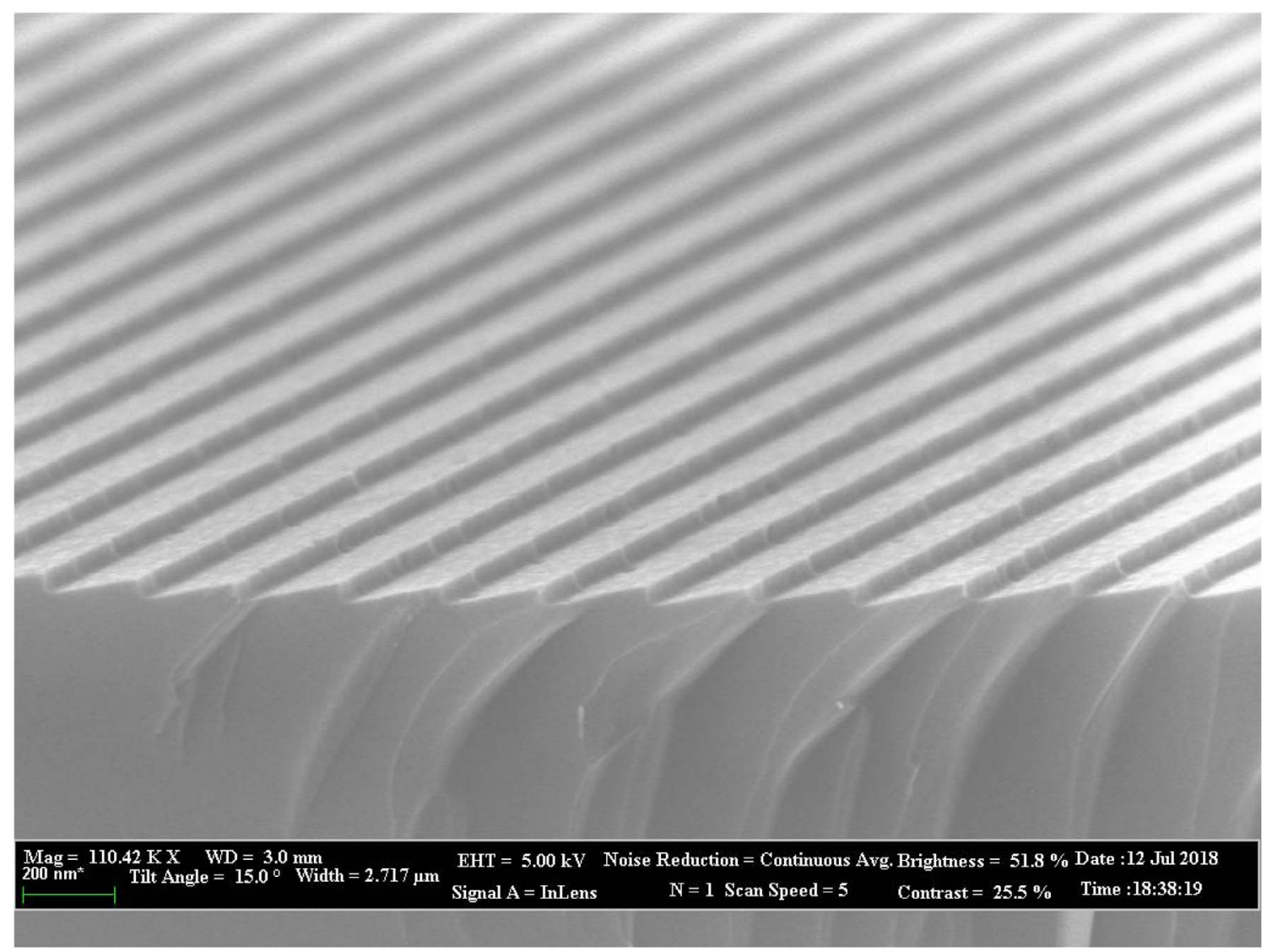

Fig. 8. Tilted scanning electron microscope image for a cleaved sample of a 5000 lines/mm blazed grating after 9 cycles of SC-I (5:1:1 DI: $\mathrm{H}_{2} \mathrm{O}_{2}: \mathrm{NH}_{4} \mathrm{OH}$ ) and HF etching. The surface of each plane is smooth, and the etch is complete. (Photo courtesy Izentis LLC).

and (3) finding the optimal layer thickness assuming top-layer oxidation.

We calculated the 2nd-order grating efficiency for 19 different coating materials. The calculations assumed singlelayer coatings of equal thickness without an adhesion layer between the coating and substrate. Three coating materials $\mathrm{Mo}, \mathrm{Ru}, \mathrm{Zr}$ - were predicted to be the most efficient. We ultimately selected $\mathrm{Zr}$ as the coating material since $\mathrm{Ru}$ is exceptionally rare and not well proven as effective in EUV optics, and Mo is susceptible to oxidation over the timescale of years (Underwood et al., 1993).

Next, we tested $\mathrm{Zr}$ paired with $\mathrm{Al}$ in a multilayer configuration similar to that applied to the optics of the High-resolution Coronal Imager (Hi-C, Kobayashi et al., 2014). Although the addition of more $\mathrm{Al} / \mathrm{Zr}$ bilayers improved the overall reflectance of the grating, these enhancements were primarily manifested in the specular 0th-order efficiency, while the 2nd-order efficiency diminished with the additions. As a result, we chose to proceed with a single-layer configuration.

Finally, we predicted the effects of top-layer oxidation and determined the optimal coating thickness. The most common oxidation product of $\mathrm{Zr}$ is $\mathrm{ZrO}_{2}$ which has a similar index of refraction to $\mathrm{Zr}$ in the EUV (Henke et al., 1993). This gives $\mathrm{Zr}$ a marked advantage over other coating materials in an oxygenrich environment. However, the addition of top-layer oxidation will inevitably change the optical properties of the grating, so it becomes critical to determine the optimal layer thickness of
$\mathrm{Zr}$ to counteract any of these ill effects. We found this thickness to be $88 \AA$ of $\mathrm{Zr}$, where the top $10 \AA$ are allowed to freely oxidize (Lyapin et al., 2004). We also tested a second configuration which capped the $88 \AA$ of $\mathrm{Zr}$ with $241 \AA$ of $\mathrm{Al}$, the top $40 \AA$ of which are allowed to oxidize primarily to $\mathrm{Al}_{2} \mathrm{O}_{3}$ (Campbell et al., 1999). The idea was to migrate the oxidation from the primary reflective substance to one that largely transmits EUV radiation. Figure 9 shows the predicted 2nd-order efficiencies for both configurations across the proposed COSIE-S passband. The configuration with the Al cap shows improvements over single-layer $\mathrm{Zr}$ at shorter wavelengths; however, it under-performs single-layer $\mathrm{Zr}$ at longer wavelengths. It should be noted that our calculations do not account for surface roughness, so our predicted efficiencies represent upper limits.

\section{Discussion}

Wide-field EUV observations can determine the start time of $\mathrm{CME}$, propagation velocity of $\mathrm{CME}$, latitude of center of CME source region, longitude of center of $\mathrm{CME}$ source region, half-width of CME, density of CME, temperature of CME. Near simultaneous wide-field EUV imaging and slitless spectroscopy can thus provide critical observational data needed for solar wind modeling as well as CME dynamics and heating in the transition corona. 


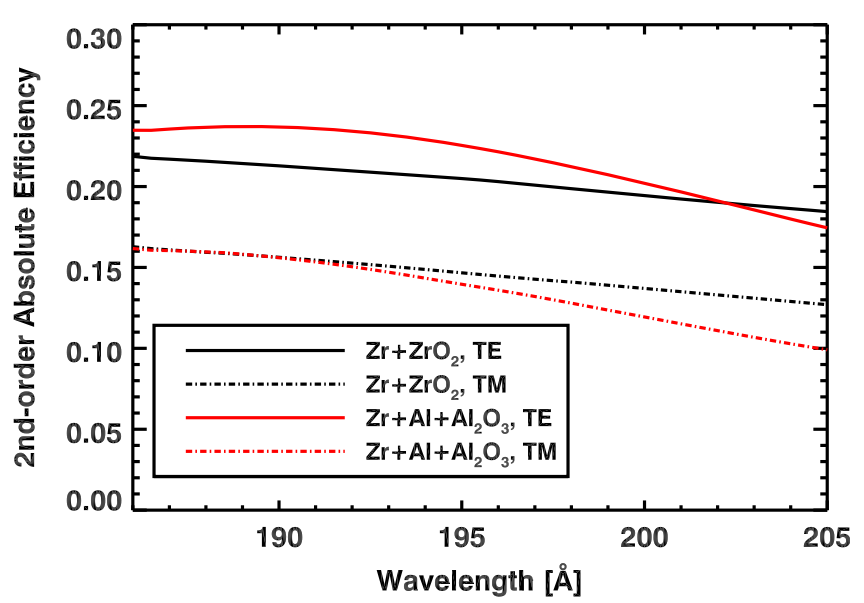

Fig. 9. Modelled 2nd-order absolute grating efficiency for the COSIE blazed reflection grating. We calculated efficiencies assuming two coating configurations: $78 \AA$ of $\mathrm{Zr}$ with a $10 \AA$ oxidation layer (black), and $88 \AA$ of $\mathrm{Zr}$ capped by $201 \AA$ of $\mathrm{Al}$ and a $40 \AA$ oxidation layer (red). We also considered two polarization states of the incident beam: transverse electric (TE, solid) and transverse magnetic (TM, dot-dash).

The instrumentation presented here has a high technology readiness level and fits within the mass, volume, power and telemetry constraints of a baseline L5 mission configuration. The data obtained will:

1. improve our understanding of the coronal conditions that control the opening and closing of the corona to the heliosphere and consequent solar wind streams, and

2. improve our understanding of the physical processes that control the early evolution of CMEs and the formation of shocks, from the solar surface out to beyond the nominal coronal source surface.

Wide-field EUV imaging will follow the evolution of the open field from eruptive timescales (minutes) to convective timescales (months). Our ability to tie specific heliospheric events back to their solar origins depends on modeling that is constrained by time-resolved observations of large-scale structures and eruptive events in the transition corona. Corresponding imaging and spectroscopy on the disk will improve the crucial initial-condition parameter inputs for space weather forecasting models, allowing for more accurate prediction of geoeffective events.

Acknowledgements. This work was supported in part by a Technology Development Grant from NASA, technical monitor J. Dan Moses, and by a NASA-HTIDS grant to GSFC, Adrian Daw PI. Roger Brissenden of SAO provided internal support that enabled development of the COSIE scientific and technical plans. We acknowledge David Broadway of MSFC, Erik Gullikson of LBL and David Windt of RXO LLC for their contributions to this program. The editor thanks two anonymous reviewers for their assistance in evaluating this paper.

\section{References}

Abbo L, Ofman L, Antiochos SK, Hansteen VH, Harra L, et al. 2016. Slow solar wind: Observations and modeling. Space Sci Rev 201: 55-108. https://doi.org/10.1007/s11214-016-0264-1.

Alzate N, Morgan H. 2017. Identification of low coronal sources of "stealth" coronal mass ejections using new image processing techniques. Astrophys J 840: 103. https://doi.org/10.3847/15384357/aa6caa.

Antiochos SK, Mikić Z, Titov VS, Lionello R, Linker JA. 2011. A model for the sources of the slow solar wind. Astrophys $J \mathbf{7 3 1}$ : 112. https://doi.org/10.1088/0004-637X/731/2/112.

Barbee TW. 1985. Multilayers for X-ray optics. Proc SPIE 563: 2-29. https://doi.org/10.1117/12.949647.

Battarbee M, Vainio R, Laitinen T, Hietala H. 2013. Injection of thermal and suprathermal seed particles into coronal shocks of varying obliquity. $A \& A$ 558: A110. https://doi.org/10.1051/00046361/201321348.

Bein BM, Berkebile-Stoiser S, Veronig AM, Temmer M, Muhr N, Kienreich I, Utz D, Vršnak B. 2011. Impulsive acceleration of coronal mass ejections. I. Statistics and coronal mass ejection source region characteristics. Astrophys J 738: 191. https://doi.org/ 10.1088/0004-637X/738/2/191.

Bein BM, Berkebile-Stoiser S, Veronig AM, Temmer M, Vršnak B. 2012. Impulsive acceleration of coronal mass ejections. II. Relation to soft X-ray flares and filament eruptions. Astrophys $J$ 755: 44. https://doi.org/10.1088/0004-637X/755/1/44.

Bruner ME, Haisch BM, Brown WA, Acton LW, Underwood JH. 1988. Soft X-ray images of the solar corona using normal incidence optics. $J$ Phys 49(C1): 115-118.

Campbell T, Kalia RK, Nakano A, Vashishta P. 1999. Dynamics of oxidation of aluminum nanoclusters using variable charge molecular-dynamics simulations on parallel computers. Phys Rev Lett 82: 4866-4869. https://doi.org/10.1103/PhysRevLett.82.4866.

Catura RC, Golub L. 1988. XUV multilayered optics for astrophysics. Rev Phys Appl 23: 1741-1746. https://doi.org/10.1051/ rphysap:0198800230100174100.

Chang C-H. 2004. High fidelity blazed grating replication using nanoimprint lithography. J Vac Sci Tech B Microelect Nanometer Struct 22: 3260. https://doi.org/10.1116/1.1809614.

Cheung MCM, Boerner P, Schrijver CJ, Testa P, Chen F, Peter H, Malanushenko A. 2015. Thermal diagnostics with the atmospheric imaging assembly on board the solar dynamics observatory: A validated method for differential emission measure inversions. Astrophys J 807: 143. https://doi.org/10.1088/0004-637X/807/2/ 143.

Cheung MCM, De Pontieu B, Martnez-Sykora J, Testa P, Winebarger A, et al. 2019. Multi-component decomposition of astronomical spectra by compressed sensing. Astrophys $J$ 882: 13. https://doi.org/10.3847/1538-4357/ab263d.

D’Huys E, Seaton DB, Poedts S, Berghmans D. 2014. Observational characteristics of coronal mass ejections without low-coronal signatures. Astrophys J 795: 49. https://doi.org/10.1088/0004637X/795/1/49.

De Pontieu B, Martnez-Sykora J, Testa P, Winebarger AR, Daw A, Hansteen V, Cheung MCM, Antolin P. 2020. The multi-slit approach to coronal spectroscopy with the multi-slit solar explorer (MUSE). Astrophys J 888: 3. https://doi.org/10.3847/1538-4357/ ab5b03.

DeForest CE, Matthaeus WH, Viall NM, Cranmer SR. 2016. Fading coronal structure and the onset of turbulence in the young solar wind. Astrophys J 828: 66. https://doi.org/10.3847/0004-637X/ $828 / 2 / 66$ 
Del Zanna G, Aulanier G, Klein K-L, Török T. 2011. A single picture for solar coronal outflows and radio noise storms. $A \& A$ 526: A137. https://doi.org/10.1051/0004-6361/201015231.

Del Zanna G, Raymond J, Andretta V, Telloni D, Golub L. 2018. Predicting the COSIE-C signal from the outer corona up to 3 solar radii. Astrophys J 865: 132. https://doi.org/10.3847/1538-4357/aadcf1.

Dissauer K, Veronig AM, Temmer M, Podladchikova T, Vanninathan K. 2018. On the detection of coronal dimmings and the extraction of their characteristic properties. Astrophys $J \mathbf{8 5 5}$ : 137. https://doi.org/10.3847/1538-4357/aaadb5.

Dissauer K, Veronig AM, Temmer M, Podladchikova T. 2019. Statistics of coronal dimmings associated with coronal mass ejections. II. Relationship between coronal dimmings and their associated CMEs. Astrophys J 874: 123. https://doi.org/10.3847/ 1538-4357/ab0962.

Dresing N, Gómez-Herrero R, Klassen A, Heber B, Kartavykh Y, Dröge W. 2012. The large longitudinal spread of solar energetic particles during the 17 January 2010 solar event. Sol Phys 281: 281-300. https://doi.org/10.1007/s11207-012-0049-y.

Evans RM, Opher M, Manchester WB, Gombosi TI. 2008. Alfvén profile in the lower corona: Implications for shock formation. Astrophys J 687: 1355-1362. https://doi.org/10.1086/592016.

Fisk LA. 2005. The open magnetic flux of the Sun. I. Transport by reconnections with coronal loops. Astrophys J 626: 563-573. https://doi.org/10.1086/429957.

Fisk LA, Zurbuchen TH. 2006. Distribution and properties of open magnetic flux outside of coronal holes. J Geophys Res (Space Phys) 111: A09115. https://doi.org/10.1029/2005JA011575.

Gallagher PT, Lawrence GR, Dennis BR. 2003. Rapid acceleration of a coronal mass ejection in the low corona and implications for propagation. Astrophys J Lett 588: L53-L56. https://doi.org/ 10.1086/375504.

Golub L, Pasachoff JM. 2009. The solar corona, 2nd edn. Cambridge University Press, Cambridge, UK.

Golub L, Hartquist TW, Quillen AC. 1989. Comments on the observability of coronal variations. Sol Phys 122: 245-261. https://doi.org/10.1007/BF00912995.

Golub L, Herant M, Kalata K, Lovas I, Nystrom G, Pardo F, Spiller E, Wilczynski J. 1990. Sub-arcsecond observations of the solar X-ray corona. Nature 344: 842-844. https://doi.org/10.1038/ $344842 \mathrm{a} 0$.

Gopalswamy N, Yashiro S. 2011. The strength and radial profile of the coronal magnetic field from the standoff distance of a coronal mass ejection-driven shock. Astrophys J Lett 736: L17. https://doi. org/10.1088/2041-8205/736/1/L17.

Goray LI. 2005. Numerical analysis of the efficiency of multilayercoated gratings using integral method. Nucl Instr Meth Phys Res A 536: 211-221. https://doi.org/10.1016/j.nima.2004.07.173.

Goryaev F, Slemzin V, Vainshtein L, Williams DR. 2014. Study of extreme-ultraviolet emission and properties of a coronal streamer from PROBA2/SWAP, Hinode/EIS and Mauna Loa Mk4 observations. Astrophys J 781: 100. https://doi.org/10.1088/0004-637X/ 781/2/100.

Haélbich RP, Kunz C. 1976. Multilayer interference mirrors for the XUV range around $100 \mathrm{eV}$ photon energy. Opt Commun 17: 287-292. https://doi.org/10.1016/0030-4018(76)90262-5.

Hannah IG, Kontar EP. 2013. Multi-thermal dynamics and energetics of a coronal mass ejection in the low solar atmosphere. $A \& A \mathbf{5 5 3}$ : A10. https://doi.org/10.1051/0004-6361/201219727.

Henke BL, Gullikson EM, Davis JC. 1993. X-ray interactions: Photoabsorption, scattering, transmission, and reflection at $\mathrm{E}=$ 50-30,000 eV, Z = 1-92. ADNDT 54: 181-342. https://doi.org/ 10.1006/adnd.1993.1013.
Hinterreiter J, Magdalenic J, Temmer M, Verbeke C, Jebaraj IC, et al. 2019. Assessing the performance of EUHFORIA modeling the background solar wind. Sol Phys 294: 170. https://doi.org/ 10.1007/s11207-019-1558-8.

Hochedez J-F, Zhukov A, Robbrecht E, van der Linden R, Berghmans D, Vanlommel P, Theissen A, Clette F. 2005. Solar weather monitoring. Ann Geophys 23: 3149-3161. https://doi.org/ 10.5194/angeo-23-3149-2005.

Kobayashi K, Cirtain J, Winebarger AR, Korreck K, Golub L, et al. 2014. The high-resolution coronal imager (Hi-C). Sol Phys 289: 4393-4412. https://doi.org/10.1007/s11207-014-0544-4.

Kozarev KA, Evans RM, Schwadron NA, Dayeh MA, Opher M, Korreck KE, Van der Holst B. 2013. Global numerical modeling of energetic proton acceleration in a coronal mass ejection traveling through the solar corona. Astrophys $J$ 778: 43. https://doi.org/10.1088/0004-637X/778/1/43.

Kozarev KA, Raymond JC, Lobzin VV, Hammer M. 2015. Properties of a coronal shock wave as a driver of early SEP acceleration. Astrophys J 799: 167. https://doi.org/10.1088/0004637X/799/2/167.

Lyapin A, Jeurgens LPH, Graat PCJ, Mittemeijer EJ. 2004. The initial, thermal oxidation of zirconium at room temperature. $J$ Appl Phys 96: 7126-7135. https://doi.org/10.1063/1.1809773.

Ma S, Attrill GDR, Golub L, Lin J. 2010. Statistical study of coronal mass ejections with and without distinct low coronal signatures. Astrophys J 722: 289-301. https://doi.org/10.1088/0004-637X/ 722/1/289.

Ma S, Raymond JC, Golub L, Lin J, Chen H, Grigis P, Testa P, Long D. 2011. Observations and interpretation of a low coronal shock wave observed in the EUV by the SDO/AIA. Astrophys $J$ 738: 160 . https://doi.org/10.1088/0004-637X/738/2/160.

Masson S, McCauley P, Golub L, Reeves KK, DeLuca EE. 2014. Dynamics of the transition corona. Astrophys $J$ 787: 145. https://doi.org/10.1088/0004-637X/787/2/145.

McComas DJ, Velli M, Lewis WS, Acton LW, Balat-Pichelin M, et al. 2007. Understanding coronal heating and solar wind acceleration: Case for in situ near-Sun measurements. Rev Geophys 45: RG1004. https://doi.org/10.1029/2006RG000195.

Mishra SK, Srivastava AK. 2019. Linkage of geoeffective stealth CMEs associated with the eruption of coronal plasma channel and jet-like structure. Sol Phys 294: 169. https://doi.org/10.1007/ s11207-019-1560-1.

Mozer FS, Agapitov OV, Bale SD, Bonnell JW, Case T, et al. 2020. Switchbacks in the solar magnetic field: Their evolution, their content, and their effects on the plasma. Astrophys J Suppl Ser 246: 68. https://doi.org/10.3847/1538-4365/ab7196.

Newkirk G, Altschuler MD, Harvey J. 1968. Influence of magnetic fields on the structure of the solar corona. In: Structure and development of solar active regions, Kiepenheuer KO (Ed.), (IAU Symposium 35), Springer, Dordrecht, p. 379.

Pontin DI, Wyper PF. 2015. The effect of reconnection on the structure of the Sun's open-closed flux boundary. Astrophys $J$ 805: 39. https://doi.org/10.1088/0004-637X/805/1/39.

Reames DV. 1999. Particle acceleration at the Sun and in the heliosphere. Space Sci Rev 90: 413-491. https://doi.org/10.1023/ A:1005105831781.

Reeves KK, Golub L. 2011. Atmospheric imaging assembly observations of hot flare plasma. Astrophys J Lett 727: L52. https://doi.org/10.1088/2041-8205/727/2/L52.

Riley P, Lionello R, Linker JA, Mikic Z, Luhmann J, Wijaya J. 2011. Global MHD modeling of the solar corona and inner heliosphere for the whole heliosphere interval. Sol Phys 274: 361-377. https://doi.org/10.1007/s11207-010-9698-x. 
Riley P, Mays ML, Andries J, Amerstorfer T, Biesecker D, et al. 2018. Forecasting the arrival time of coronal mass ejections: analysis of the CCMC CME scoreboard. Space Weather 16: 12451260. https://doi.org/10.1029/2018SW001962.

Robbrecht E, Patsourakos S, Vourlidas A. 2009. No trace left behind: STEREO observation of a coronal mass ejection without low coronal signatures. Astrophys J 701: 283-291. https://doi.org/ 10.1088/0004-637X/701/1/283.

Robbrecht E, Wang Y-M. 2010. The temperature-dependent nature of coronal dimmings. Astrophys J Lett 720: L88-L92. https://doi. org/10.1088/2041-8205/720/1/L88.

Savage SL, McKenzie DE, Reeves KK, Forbes TG, Longcope DW. 2010. Reconnection outflows and current sheet observed with Hinode/XRT in the 2008 April 9 "Cartwheel CME" flare. Astrophys J 722: 329-342. https://doi.org/10.1088/0004-637X/722/1/329.

Savage SL, Holman G, Reeves KK, Seaton DB, McKenzie DE, Su Y. 2012a. Low-altitude reconnection inflow-outflow observations during a 2010 November 3 solar eruption. Astrophys $J$ 754: 13. https://doi.org/10.1088/0004-637X/754/1/13.

Savage SL, McKenzie DE, Reeves KK. 2012b. Re-interpretation of supra-arcade downflows in solar flares. Astrophys J Lett 747: L40. https://doi.org/10.1088/2041-8205/747/2/L40.

Seaton DB, De Groof A, Shearer P, Berghmans D, Nicula B. 2013. SWAP observations of the long-term, large-scale evolution of the extreme-ultraviolet solar corona. Astrophys $J$ 777: 72 . https://doi. org/10.1088/0004-637X/777/1/72.

Sheeley NR. 2017. Origin of the Wang-Sheeley-Arge solar wind model. Hist Geo Space Sci 8: 21-28. https://doi.org/10.5194/hgss8-21-2017.

Sheeley NR, Wang Y-M. 2007. In/out pairs and the detachment of coronal streamers. Astrophys J 655: 1142-1156. https://doi.org/ $10.1086 / 510323$.

Silk JK, Kahler S, Krieger AS, Timothy AF, Vaiana GS. 1975. Objective grating studies of X-ray flare spectra. Osservazioni $e$ Memorie dell'Osservatorio Astrofisico di Arcetri 104: 143-156.

Slemzin V, Bougaenko O, Ignatiev A, Kuzin S, Mitrofanov A, Pertsov A, Zhitnik I. 2008. Off-limb EUV observations of the solar corona and transients with the CORONAS-F/SPIRIT telescopecoronagraph. Ann Geophys 26: 3007-3016. https://doi.org/ 10.5194/angeo-26-3007-2008.

Spiller E. 1974. Multilayer interference coatings for the vacuum ultraviolet. In: Space optics, Thompson BJ, Shannon RR (Eds.), National Academy of Sciences, Washington, DC, pp. 570-581.

Sterling AC, Hudson HS. 1997. Yohkoh SXT observations of X-ray "dimming" associated with a halo coronal mass ejection. Astrophys J 491: L55-L58. https://doi.org/10.1086/311043.

Su Y, Veronig AM, Holman GD, Dennis BR, Wang T, Temmer M, Gan W. 2013. Imaging coronal magnetic-field reconnection in a solar flare. Nat Phys 9: 489-493. https://doi.org/10.1038/nphys2675.

Su Y, van Ballegooijen A, McCauley P, Ji H, Reeves KK, DeLuca EE. 2015. Magnetic structure and dynamics of the erupting solar polar crown prominence on 2012 March 12. Astrophys J 807: 144. https://doi.org/10.1088/0004-637X/807/2/144.

Sun JQ, Cheng X, Ding MD, Guo Y, Priest ER, Parnell CE, Edwards SJ, Zhang J, Chen PF, Fang C. 2015. Extreme ultraviolet imaging of three-dimensional magnetic reconnection in a solar eruption. Nat Commun 6: 7598. https://doi.org/10.1038/ncomms8598.

Tadikonda SK, Freesland DC, Minor RR, Seaton DB, Comeyne GJ, Krimchansky A. 2019. Coronal imaging with the solar ultraviolet imager. Sol Phys 294: 28. https://doi.org/10.1007/s11207-0191411-0.

Temmer M. 2016. Kinematical properties of coronal mass ejections. Astron Nachr 337: 1010. https://doi.org/10.1002/asna.201612425.
Thompson BJ, Cliver EW, Nitta N, Delannée C, Delaboudinière J-P. 2000. Coronal dimmings and energetic CMEs in April-May 1998. Geophys Res Lett 27: 1431-1434. https://doi.org/10.1029/ 1999GL003668.

Tousey R, Bartoe JDF, Bohlin JD, Brueckner GE, Purcell JD, Scherrer VE, Sheeley NR Jr, Schumacher RJ, Vanhoosier ME. 1973. A preliminary study of the extreme ultraviolet spectroheliograms from Skylab. Sol Phys 33: 265. https://doi.org/10.1007/ BF00152418.

Underwood JH, Gullikson EM, Nguyen K. 1993. Tarnishing of Mo/ Si multilayer X-ray mirrors. Appl Opt 32: 6985. https://doi.org/ 10.1364/AO.32.006985.

Vásquez AM, van Ballegooijen AA, Raymond JC. 2003. The effect of proton temperature anisotropy on the solar minimum corona and wind. Astrophys J 598: 1361. https://doi.org/10.1086/ 379008.

Veronig AM, Muhr N, Kienreich IW, Temmer M, Vršnak B. 2010. First observations of a dome-shaped large-scale coronal extremeultraviolet wave. Astrophys J Lett 716: L57-L62. https://doi.org/ 10.1088/2041-8205/716/1/L57.

Veronig AM, Podladchikova T, Dissauer K, Temmer M, Seaton DB, Long D, Guo J, Vršnak B, Harra L, Kliem B. 2018. Genesis and impulsive evolution of the 2017 September 10 coronal mass ejection. Astrophys J 868: 107. https://doi.org/10.3847/1538-4357/ aaeac5.

Veronig AM, Gömöry P, Dissauer K, Temmer M, Vanninathan K. 2019. Spectroscopy and differential emission measure diagnostics of a coronal dimming associated with a fast halo CME. Astrophys J 879: 85. https://doi.org/10.3847/1538-4357/ab2712.

Walker ABC, Barbee TW, Hoover RB, Lindblom JF. 1988. Soft $\mathrm{X}$-ray images of the solar corona with a normal-incidence cassegrain multilayer telescope. Science 241: 1781-1787. https://doi.org/10.1126/science.241.4874.1781.

Walker ABC, Hoover RB, Barbee TW Jr. 1993. High resolution thermally differentiated images of the chromosphere and corona. In: Physics of solar and stellar coronae, Linsky JF, Serio S (Eds.), Astrophysics and Space Science Library, Springer, Dordrecht, Vol. 183, pp. 83-96. https://doi.org/10.1007/978-94-011-1964-1_7.

Wang Y-M. 1996. Nonradial coronal streamers. Astrophys J Lett 456: L119. https://doi.org/10.1086/309871.

Weberg MJ, Lepri ST, Zurbuchen TH. 2015. Coronal sources, elemental fractionation, and release mechanisms of heavy ion dropouts in the solar wind. Astrophys $J$ 801: 99. https://doi.org/ 10.1088/0004-637X/801/2/99.

West MJ, Seaton DB. 2015. SWAP observations of post-flare giant arches in the long-duration 14 October 2014 solar eruption. Astrophys J Lett 801: L6. https://doi.org/10.1088/2041-8205/801/ 1/L6.

Winebarger AR, Weber M, Bethge C, Downs C, Golub L, et al. 2019. Unfolding overlapped slitless imaging spectrometer data for extended sources. Astrophys J 882: 12. https://doi.org/10.3847/ 1538-4357/ab21db.

Yan XL, Yang LH, Xue ZK, Mei ZX, Kong DF, Wang JC, Li QL. 2018. Simultaneous observation of a flux rope eruption and magnetic reconnection during an X-class solar flare. Astrophys $J$ Lett 853: L18. https://doi.org/10.3847/2041-8213/aaa6c2.

Yeates AR, Mackay DH, van Ballegooijen AA. 2008. Modelling the global solar corona II: Coronal evolution and filament chirality comparison. Sol Phys 247: 103. https://doi.org/10.1007/s11207007-9097-0.

Yokoyama T, Akita K, Morimoto T, Inoue K, Newmark J. 2001. Clear evidence of reconnection inflow of a solar flare. Astrophys $J$ Lett 546: L69-L72. https://doi.org/10.1086/318053. 
Zhang J, Dere KP, Howard RA, Kundu MR, White SM. 2001. On the temporal relationship between coronal mass ejections and flares. Astrophys J 559: 452-462. https://doi.org/10.1086/322405.

Zhitnik IA, Ignatiev AP, Korneev VV, Krutov VV, Kuzin SV, et al. 1998. Instruments for imaging XUV spectroscopy of the sun on board the CORONAS-I satellite. Proc SPIE 3406: 1-19. https://doi.org/10.1117/12.310979.
Zucca P, Pick M, Démoulin P, Kerdraon A, Lecacheux A, Gallagher PT. 2014. Understanding coronal mass ejections and associated shocks in the solar corona by merging multiwavelength observations. Astrophys J 95: 68. https://doi.org/10.1088/0004-637X/795/ $1 / 68$.

Cite this article as: Golub L, Cheimets P, DeLuca EE, Madsen CC, Reeves KK 2020. EUV imaging and spectroscopy for improved space weather forecasting. J. Space Weather Space Clim. 10, 37. https://doi.org/10.1051/swsc/2020040. 